\title{
Manejo endoscópico de la obesidad
}

\author{
Sergio Solana-Sentíes* \\ Hospital Lomas de San Luis, San Luis Potosí, México
}

Palabras clave: Balón intragástrico. Porcentaje de pérdida de peso corporal total. Porcentaje de pérdida de exceso de peso. Gastroplastia en manga.

Actualmente la mayoría de los métodos endoscópicos para el manejo de la obesidad son restrictivos, destacando principalmente el balón intragástrico y la cirugía endoscópica a través de un dispositivo denominado endostich, que permite según el tipo de procedimiento la remodelación gástrica modificando su anatomía y fisiología, generando cambios en el acomodo de los alimentos y modificando el tiempo de vaciamiento gástrico lo cual contribuye a la sensación de saciedad.

Ana Paula S. Kotinda et al..$^{1}$ realizaron un estudio para evaluar la eficacia de los balones intragástricos (IGB) disponibles actualmente, en comparación con solo intervenciones en el estilo de vida para la pérdida de peso en pacientes con sobrepeso y obesidad. Solo ensayos controlados aleatorizados (ECA), que compararon IGB con o sin intervención del estudio de vida (grupo de estudio) y simulación o intervención del estilo de vida (grupo control) fueron incluidos. Un total de 13 ECA con 1523 pacientes (859 en el grupo de intervención y 664 en el grupo control) se incluyeron en el metaanálisis. La diferencia en la media del \% EWL y \% TWL en el seguimiento fue $17.98 \%$ y $4.40 \%$ respectivamente. Los autores concluyen que la terapia con IGB es más efectiva que solo la intervención en el estilo de vida para la pérdida de peso.
Veeravich Jaruvongvanich et al. ${ }^{2}$ realizaron un estudio prospectivo cuyo objetivo fue investigar el papel de los factores fisiológicos basales para la pérdida de peso y secundariamente evaluar los efectos del ajuste de volumen del balón ajustable en el vaciamiento gástrico y su relación con la pérdida de peso.

Se incluyeron cuarenta pacientes en quienes se colocó un IGB tipo Orbera a 28 pacientes con un volumen de llenado de $600 \mathrm{ml}$. y spatz a 12 pacientes con un volumen de $550 \mathrm{ml}$. y a los 4 meses, ajustando el volumen final medio a $850 \mathrm{ml}(\mathrm{n}=10)$. Se determinó el tiempo de vaciamiento gástrico al inicio y a los 3 meses, y adicionalmente en el grupo de spatz, una prueba de vaciamiento gástrico adicional 1 mes después del ajuste, expresándose como tiempo de vaciamiento al $50 \%$ (T50). El \% medio de pérdida de peso corporal total (\%TBWL) a los 3 y a los 6 meses fue de $10.6 \pm$ 4.2 y $13.9 \pm 6.8$, respectivamente. T50 se incrementó a los 3 meses con un cambio medio de T50 de 71.9 minutos. En el grupo de Orbera se encontró una correlación significativa del volumen de llenado basal > 600 $\mathrm{ml}(p=0.028)$ y cambios en el T50 a tres meses del basal $(p=0.008)$, y al $\%$ de pérdida de peso corporal total a los 6 meses. El ajuste en el volumen del balón a los 4 meses de 250-300 ml. en el grupo de spatz resulto en un mayor retraso del tiempo de vaciamiento

\section{Correspondencia:}


gástrico y un incremento medio en el porcentaje de pérdida de peso corporal total de $2.2 \pm 1.6 \%$. Los autores concluyen que además del cambio en el vaciamiento gástrico, un mayor volumen basal de llenado fue significativamente asociado con una mayor pérdida de peso. Un incremento en el volumen basal del balón puede aumentar la pérdida de peso.

Actualmente la FDA en E.U. ha aprobado el uso del balón intragástrico lleno de gas (Obalon, GF-IGB) o lleno de líquido (orbera, FF-IGB)

Aymen Almuhaidbet et al..$^{3}$ realizaron un estudio comparativo entre estos dos tipos de IGB, evaluando su eficacia, tolerancia, y seguridad. Estudio de cohorte, retrospectivo entre octubre de 2015 y mayo de 2019. La decisión de recibir cualquiera de los diferentes sistemas de balón fue a discreción de los pacientes. El objetivo fue evaluar el \% de pérdida de peso corporal total (\%TBWL) y el porcentaje de pérdida de peso corporal en exceso (\%EBWL) a los 6 meses. Secundariamente se evaluaron los eventos adversos. 76 pacientes fueron sometidos a colocación de IGB, 50 pacientes recibieron GF-IGB y 26 pacientes recibieron FF-IGB. Once pacientes requirieron del retiro del balón con 20 semanas. No hubo diferencias estadísticamente significativas en \%TBWL o \%EBWL a los 6 meses entre los sistemas de IGB ( $p=0.66$ y 0.55 ). Los pacientes con FF-IGB (Orbera) tuvieron más probabilidades de tener visitas a urgencias debido a síntomas gastrointestinales ( $15 \%$ vs. $4 \%$ ). Los autores concluyen que los dos sistemas de balón gástrico aprobados por FDA fueron comparables en términos de eficacia; sin embargo, el GF-IGB (Obalon) fue más tolerable que el FF-IGB (Orbera).

Shailendra Singh et al. ${ }^{4}$ comparo individualmente el IGB y la gastroplastia endoscópica (ESG) en cuanto a su eficacia, durabilidad, y seguridad. Se realizaron búsquedas sistemáticas para investigar el uso del IGB y ESG. Se incluyeron los estudios que informaron sobre el porcentaje de pérdida de peso total (\%TWL) 0 el porcentaje de pérdida de peso excesiva (\%EWL) con al menos doce meses de seguimiento.

Se incluyeron un total de 28 estudios en el análisis final. Solo uno comparó directamente ESG con IGB, 9 estudios evaluaron ESG solo, mientras que 18 estudios evaluaron IGB. A los 12 meses de seguimiento después del ESG, la media del $\%$ TWL fue de 17.51 , y el $\%$ EWL fue de 60.51. La media de \% TWL y \% EWL después del IGB a los 12 meses fue de 10.35 y 29.65, respectivamente. La media del \% TWL y \% EWL después del retiro del IGB, disminuyeron significativamente a los 18 -24 meses. Se observaron efectos adversos graves en $<5 \%$. Este estudio sugiere que los resultados de ESG ofrecen una pérdida de peso mayor y más sostenida que el IGB.

Debashis Reja et al. ${ }^{5}$ realizaron un estudio retrospectivo internacional, multicéntrico de pacientes consecutivos en 4 cetros que se sometieron a ESG entre junio-2016 y septiembre de 2019, cuyo objetivo primario fue evaluar el resultado metabólico mediante la tensión arterial sistólica y diastólica media, enzimas hepáticas, perfil de lípidos, el IMC, el peso y la A1C. Incluyeron a 92 pacientes, edad media de 43.2 años. Se observó a los 2.4 meses una reducción significativa de la presión sistólica en $11 \mathrm{mmHg}$ $(p<0.00003)$ la presión arterial diastólica en 4.65 $(p<0.004)$, peso en 24 libras $(p<0.00001)$, IMC en $4.1 \mathrm{~kg} / \mathrm{m} 2(p<0.00001)$ y ALT en $8.62 \mathrm{U} .(p<0.004)$. Los autores concluyen que la ESG resulto en una mejoría en presión sanguínea, daño hepático y una pérdida de peso significativa, en la insulina, medicamentos antihipertensivos y para reflujo gastro-esofágico, demostrando un impacto importante en la pérdida de peso, pero también en las enfermedades metabólicas.

La diabetes mellitus es la 7.- causa de muerte a nivel mundial de acuerdo con la OMS. La enfermedad hepática grasa no alcohólica (EGHNA) está asociada con diabetes y obesidad. El duodeno tiene un papel importante en la absorción nutricia y metabolismo de glucosa. La remodelación de la mucosa duodenal (DMR) es una nueva técnica endoscópica de ablación hidrotérmica de la mucosa que potencialmente mejora el control glucémico.

Neil Bhogal et al. ${ }^{6}$ realizaron una revisión sistemática y meta análisis evaluando la eficacia y seguridad de la remodelación de la mucosa duodenal (duodenal mucosal resurfacing DMR) para diabetes y enfermedad por hígado graso no alcohólico. Se realizo una búsqueda en varias bases de datos hasta octubre de 2019. El resultado primario fue el cambio en los niveles séricos de $\mathrm{HbA1c}$. Los resultados secundarios incluyeron el cambio en la ALT y el peso así como efectos adversos

Se incluyeron un total de seis brazos de estudio para el análisis de un total de 181 pacientes. Todos los estudios fueron prospectivos. Cuatro estudios reportaron cambios en la HbA1c a los 12 meses y dos estudios lo reportaron a los 3 meses. En general la disminución media en HgbA1c fue de 0.94\% (0.69-1.19). Hubo seis eventos adversos. Hubo una disminución media de ALT de 9.04 y una disminución media en el peso de $2.7 \mathrm{~kg}$ 
Los autores concluyen que la DMR es una técnica segura y eficaz para mejorar el control glucémico, enzimas hepáticas y peso. DMS deberá ser considerado en el manejo de diabetes mellitus y EHGNA.

\section{Financiamiento}

Los autores no recibieron patrocinio para llevar a cabo este artículo.

\section{Conflicto de intereses}

Los autores declaran no tener conflicto de intereses alguno.

\section{Bibliografía}

1. Ana Paula S. Kotinda, Wanderlei M. Bernardo, Alberto M. da Ponte et al. Efficacy of intragastric balloons for weight loss in overweigth and obese adults: A systematic review and meta-analysis of randomized controlled trials Abstract Sa 1967 DDW 2020.

2. Veeravich Jaruvongvanich, Gontrand Lopez-Nava, Andrew C Storm, et al. Baseline physiologic predictors of weight loss after intragastric balloon placement: A multicenter prospective cohort. Abstract Sa 1934 DDW 2020

3. Aymen Almuhaidb, Eric Swei, Abdullah Al-Shahrani, et al. Comparison of the efficacy and saftey of the FDA-Approved intragastric balloon systems in a clinical setting. Abstract Sa 1955.

4. Shailendra singh, Diogo T de Moura, Ahmad Khan, et al. Intragastric balloon versus endoscopic sleeve gastroplasty for the treatment of obesity: A systematic review and meta-analysis. Abstract Sa 1946.

5. Debashis Reja, Haroon M Shahid, Amy Tyberg, et al. Weight and metabolic outcomes associated with endoscopic sleeve gastroplasty: A multicenter study. Abstract Sa 1951

6. Neil Bhogal, Harian Sayles, Saurabh et al. Efficcacy and safety of duodenal mucosal resurfacing for diabetes and non-alcoholic fatty liver disease: A systematic review and meta-analisis. Abstract Sa1950 DDW 2020. 